INRA Prod. Anim., 1997, 10 (1), 111-119

\section{N. BARRE}

CIRAD-EMVT BP 515, 97165 Pointe-à-Pitre, Guadeloupe (FWI)
Les tiques des ruminants dans les Petites Antilles : biologie, importance économique, principes de lutte

\footnotetext{
Deux espèces de tiques infestent les ruminants aux Antilles. La première transmet la cowdriose et favorise l'apparition de la dermatophilose, deux graves maladies des bovins de génotypes d'introduction récente et des caprins. Du fait de son importance économique et de son aptitude à s'étendre vers des pays voisins encore indemnes, et compte tenu de sa sensibilité aux acaricides, son éradication est recommandée. L'autre espèce est déjà cosmopolite dans la région, mais constitue un vecteur de moindre importance. Elle manifeste une résistance aux acaricides et ne peut être raisonnablement combattue que par les procédés classiques.
}

\begin{abstract}
Résumé
Deux espèces de tiques tropicales, Boophilus microplus, d'origine asiatique, et Amblyomma variegatum, d'origine africaine, parasitent les ruminants des Antilles, en particulier des Antilles françaises (Guadeloupe et Martinique). Elles sont vectrices de diverses maladies : babésioses et anaplasmose pour Boophilus, theilérioses bénignes et cowdriose pour Amblyomma. De plus Amblyomma est associé aux formes cliniques sévères, fréquemment mortelles, de la dermatophilose. En Guadeloupe, où la majorité des bovins sont de type créole, hautement résistants à ces maladies, mais où les caprins paient un lourd tribut à la cowdriose, le préjudice annuel généré par les tiques est estimé à 13,8 MF. Alors que Boophilus a couvert dans les Antilles et sur le continent l'ensemble de son aire de distribution potentielle, Amblyomma, l'espèce la plus pathogène, n'est encore présente que dans les Petites Antilles et à Porto Rico. Encore confinée à 4 îles au milieu de ce siècle, elle a infesté 14 îles nouvelles au cours des 25 dernières années. De fortes présomptions permettent de penser que le Héron garde-bœufs, arrivé dans les Petites Antilles à la fin des années 50 depuis l'Afrique, largement répandu dans la Caraïbe, pourrait être à l'origine de l'accélération récente de la propagation de la tique dans la région. Celle-ci menace le continent américain et les Grandes Antilles. Malgré de grandes capacités de diffusion régionale sur ses hôtes, cette tique a une productivité numérique, donc un pouvoir d'installation et de colonisation, bien moindre que Boophilus. Dès l'apparition d'un foyer, la mise en place de mesures adaptées peut donc efficacement entraver son développement. Là où elle est établie, des mesures rigoureuses doivent permettre de rompre son cycle de développement et de détruire définitivement ses populations. De plus, elle ne semble pas manifester actuellement de phénomène de résistance aux acaricides, et elle est, au stade adulte, assez spécifique des animaux domestiques de grande taille. Ceci est en faveur de la mise en ouvre d'une stratégie de lutte ayant pour objectif un programme d'éradication spécifique basé sur l'application régulière d'acaricides sur ses hôtes domestiques.
\end{abstract}

Les tiques sont des arthropodes appartenant à la classe des Arachnides et qui se nourrissent du sang de leur hôte au cours des phases parasitaires. Les tiques ont d'abord évolué sur les reptiles au cours du paléozoïque et du mésozoïque. Avec la diversification des vertébrés survenue lors des époques géologiques ultérieures, elles se sont adaptées à des hôtes nouveaux pour devenir des parasites spécifiques de certaines familles d'oiseaux ou de mammifères en plus de leurs hôtes originels. On compte actuellement 800 espèces de tiques dont 80 environ ont largement profité des aménagements agricoles et de la domestication animale. Ces activités humaines ont accru la densité des hôtes potentiels dans les zones d'élevage ce qui a eu pour effet de faciliter la rencontre hôtes-parasites et le déroulement des cycles parasitaires. Il en est résulté une explosion de certaines espèces dont l'impact économique est devenu incompatible avec les objectifs de rentabilité des élevages. En effet, à l'action pathogène directe liée à la fixation du rostre dans la peau pendant les 5 à 25 jours que dure le repas de sang, s'ajoute, pour plusieurs espèces, la capacité de transmission d'agents pathogènes à l'hôte lors de cette fixation. La situation est particulièrement préoccupante 
dans les zones tropicales où la diversité des espèces de tiques parasites du bétail va de pair avec celle des maladies transmises.

Les mouvements humains et ceux des animaux domestiques au cours des derniers siècles ont largement contribué à la diffusion mondiale des espèces les mieux adaptées aux animaux domestiques, restées jusqu'alors limitées à l'aire d'extension de leurs hôtes sauvages. Les Antilles n'ont pas échappé à cette règle et sont maintenant confrontées à des situations sanitaires fortement dominées par les tiques et les maladies qu'elles transmettent.

\section{1 / Présentation du milieu}

\section{1 / Les Antilles : contexte biogéographique et agronomique}

Les Petites Antilles forment un chapelet d'îles situées entre $12^{\circ}$ et $19^{\circ}$ de latitude nord et entre $59^{\circ}$ et $65^{\circ}$ de longitude ouest, qui bénéficient d'un climat chaud et humide toute l'année ( 23 à $29{ }^{\circ} \mathrm{C}$ au niveau de la mer selon le mois, 1200 à $4500 \mathrm{~mm}$ de pluies annuelles selon la région). Le climat est plus sec de janvier à juin durant le "carême » et plus humide de juillet à décembre pendant l'" hivernage ». Guadeloupe et Martinique sont au centre de l'arc antillais, la Guadeloupe étant, avec ses 5 dépendances, la plus grande des îles des Petites Antilles (1 $700 \mathrm{~km}^{2}$ ). Depuis l'arrivée des premiers colons au $17^{\mathrm{e}}$ siècle, les Antilles françaises ont entretenu un commerce prospère avec la métropole et ses autres colonies. C'est d'Afrique par exemple qu'est originaire le bétail de ces îles, mais les croisements récents sont réalisés essentiellement à partir de races métropolitaines améliorées. Les effectifs estimés des animaux domestiques en Guadeloupe et Martinique sont portés au tableau 1. Ces deux îles couvrent $64 \%$ de la superficie des Petites Antilles et totalisent $76 \%$ des bovins et $55 \%$ des petits ruminants de la région.

\section{2 / L'élevage et les pathologies animales}

Quelques règles président à la situation sanitaire observée dans la région.

Les îles des Petites Antilles étaient inhabitées avant l'arrivée, à partir de 5000 ans originaires des côtes vénézuéliennes. La faune des vertébrés indigènes était pauvre (chauves-souris, oiseaux, reptiles, peut-être Raton laveur et Agouti) et ne comportait pas avant notre ère, des peuples précolombiens

de grands herbivores ni de porcins susceptibles d'héberger une population parasitaire naturelle transférable ultérieurement sur des animaux domestiques importés, taxonomiquement proches. Tous les grands herbivores actuels ont été introduits. Ils ont apporté des parasites et des agents pathogènes qui leur étaient associés dans leurs zones géographiques d'origine, essentiellement l'Europe occidentale et l'Afrique.

L'insularité assure un isolement relatif formant une certaine barrière sanitaire. Il y a peu de grandes maladies infectieuses aux Antilles alors qu'elles sont pourtant présentes sur le continent américain voisin. L'isolement est efficace entre les îles elles-mêmes : la rage par exemple, qui existe à Grenade depuis la fin des années 1940 (Nellis et Everard 1983), n'a pas essaimé ailleurs dans les Petites Antilles. Cet isolement relatif a également certainement contribué à retarder la propagation des tiques dans la région, au moins jusqu'à ces dernières années. Cette situation peut être bouleversée par une intensification des échanges commerciaux sans précautions ou par un événement épidémiologique nouveau, comme l'arrivée récente du Héron garde-bœufs (Bubulcus ibis), qui a, semble til, contribué à l'accélération de la propagation de la tique Amblyomma variegatum (Uilenberg 1990, Corn et al 1993, Barré et al 1995).

Le climat tropical chaud et humide prévalant aux Antilles est favorable au développement de parasites adaptés aux régions tropicales et donc originaires de ces régions, bien plus qu'à celui de parasites des zones tempérées.

\section{3 / Espèces et races animales élevées}

En Guadeloupe, la grande majorité des bovins (90\% selon Salas et al 1988, mais plus que $72 \%$ en 1995 selon M. Naves, communication personnelle) est de race créole, animal de phénotype zébu, issu de croisements anciens entre des zébus africains et des taurins africains et européens, notamment ibériques. En Martinique, le cheptel local a en grande partie cédé la place à des races européennes à viande mais surtout à un important apport de brahmans américains.

Les caprins de Guadeloupe et Martinique, appelés " cabrits créoles ", sont de petit format, de type brevipes ; ils sont sans doute originaires d'Afrique de l'Ouest. On rencontre quelques croisés avec des anglo-nubiens. Les ovins sont quasi absents de Guadeloupe ; en Martinique ils sont de race créole, St Martin et « Black-Belly » (Tatareau et al 1991).

\section{2 / Les tiques du bétail} 1990, (2) Champanhet 1995, (3) FAO 1992, (4) Tatareau et al 1991.

\begin{tabular}{|l|c|c|c|c|}
\hline & Superficie $\left(\mathrm{km}^{2}\right)$ & Effectif bovin & Effectif caprin & Effectif ovin \\
\hline Guadeloupe & 1780 & $68500^{(1)}$ & $28900^{(4)}$ & $3800^{(4)}$ \\
Martinique & 1080 & $28200^{(2)}$ & $13100^{(4)}$ & $43700^{(4)}$ \\
Autres îles & 1560 & $29000^{(3)}$ & $66600^{(3)}$ & \\
\hline
\end{tabular}

\section{1 / Espèces présentes, origine et répartition}

Il existe une douzaine d'espèces appartenant à 6 genres (Morel 1966), qui sont indi- 
gènes et parasites des oiseaux de mer ou des chauves-souris, ou qui ont été introduites avec des animaux importés : crapaud-buffle, mangouste, rats, ruminants, chevaux, chiens, volailles. Les ruminants sont les hôtes électifs de 2 espèces d'Ixodidés, toutes deux exotiques :

- Boophilus microplus, est une tique monoxène (les 3 stades se nourrissent sur un seul et même hôte) très spécifique des bovins (monotrope). Elle est d'origine asiatique mais a sans doute été introduite aux Antilles à partir d'Amérique centrale ou australe, ce continent ayant lui même été infesté par du bétail transporté à travers le Pacifique venant d'Asie orientale ou d'Australie (Morel 1966). L'infestation des Antilles s'est faite d'île en île, sans doute précocement puisque sa dénomination de "tique créole " en Guadeloupe et Martinique lui assigne, dans la tradition populaire, une origine locale. Elle est actuellement cosmopolite dans les Petites et Grandes Antilles et en Amérique tropicale. C'est le vecteur des babésioses (Babesia bigemina et $B$. bovis), et un des vecteurs de l'anaplasmose (Anaplasma marginale).

- Amblyomma variegatum, est une tique trixène (un hôte différent pour chacun des 3 stades), à large spectre d'hôtes (télotrope), mais plus spécifique des ruminants au stade adulte (tendance au monotropisme). Elle est d'origine africaine, sans doute d'Afrique de l'ouest comme permettent de le supposer nos connaissances historiques sur les circuits commerciaux aux $17^{\mathrm{e}}$ et $18^{\mathrm{e}}$ siècles (Maillard et Maillard 1995) et son nom de " tique sénégalaise " couramment employé dans les Antilles françaises. Depuis 1830, date de son implantation supposée en Guadeloupe (Curasson 1943), elle a colonisé 18 îles des Grandes (Porto Rico) et Petites Antilles et menace maintenant le continent américain (Barré et al 1995 ; figure 1). Elle transmet la cowdriose (Cowdria ruminantium), identifiée dans trois îles des Petites Antilles, les deux theilérioses bénignes (Theileria mutans et $T$. velifera) et elle est associée aux formes cliniques sévères de dermatophilose (Dermatophilus congolensis).

\section{2 / Sensibilité comparée des ruminants aux tiques et aux maladies transmises par les tiques ou qui leurs sont associées ; impact économique}

Des observations répétées ont montré que les petits ruminants ne sont pas ou très peu infestés par la tique Boophilus, à laquelle, à la différence des zébus créoles et brahmans, les bovins européens sont très sensibles. Par contre, tous ces ruminants, quelle que soit l'espèce ou la race, sont infestés par Amblyomma (tableau 2).

Leur sensibilité aux maladies associées aux tiques (dermatophilose), ou transmises par elles, est très variable et liée à une réceptivité différente ou à une plus ou moins grande capacité à développer des réactions immunitaires efficaces. Le bovin brahman manifeste une extrême sensibilité à la dermatophilose, une maladie bactérienne cutanée à évolution lente et fréquemment mortelle, mais une grande résistance aux protozoaires sanguins Babesia bovis et $B$. bigemina et aux rickettsies Anaplasma marginale et Cowdria ruminantium, auxquels les bovins européens sont par contre très sensibles. Les petits ruminants sont sensibles à la dermatophilose et d'autant plus sensibles à la cowdriose qu'ils ont été élevés ou sont originaires d'une zone indemne d'Amblyomma.

Compte tenu de la sensibilité variable des races et espèces de ruminants aux maladies liées aux tiques, l'impact économique de ces maladies sera très différent d'une île à l'autre et d'un élevage à l'autre et dépendra des choix génétiques et zootechniques qui ont été faits.

Malgré la large domination numérique des bovins créoles en Guadeloupe, les pertes annuelles dues aux tiques et maladies transmises sont estimées à $12,6 \mathrm{MF}$ sur bovins et 1,2 MF sur petits ruminants (Camus 1987, Stachurski 1988). Celles-ci sont certainement très supérieures en Martinique où les zébus brahmans, abondants dans cette île, paient un lourd tribut à la dermatophilose.

Boophilus a donc un pouvoir pathogène direct et indirect relativement modeste sur le bétail rustique et les zébus, nul sur les petits ruminants, à la différence d'Amblyomma dont le long rostre induit plaies et abcès et qui est vecteur ou qui est associé à deux très graves maladies. Amblyomma est donc l'espèce de tique la plus néfaste à l'élevage et qui nécessite d'être combattue en priorité.

\section{3 / Biologie comparée des deux espèces de tiques. Dynamique de population}

L'analyse des aptitudes des tiques à la propagation, à l'établissement et à l'accroissement de leur population permet de prévoir leurs zones d'installation potentielles et de recommander des principes généraux de lutte adaptés à leurs capacités de multiplication et d'extension.

\section{Les bovins locaux sont peu infestés par Boophilus, alors que les bovins d'origine européenne sont infestés par les 2 tiques et très sensibles aux maladies transmises. Les petits ruminants ne sont infestés que par Amblyomma et paient un lourd tribut à la cowdriose.}

Tableau 2. Sensibilité des ruminants des Antilles aux tiques et aux maladies transmises par les tiques ou qui leurs sont associées.

\begin{tabular}{|l|c|c|c|c|}
\hline & $\begin{array}{c}\text { Bovins } \\
\text { Créoles }\end{array}$ & $\begin{array}{c}\text { Bovins } \\
\text { européens }\end{array}$ & $\begin{array}{c}\text { Bovins } \\
\text { Brahman }\end{array}$ & $\begin{array}{c}\text { Petits } \\
\text { ruminants }\end{array}$ \\
\hline Boophilus & + & +++ & + & - \\
Maladie transmise : & - & +++ & - & - \\
- babésiose & - & ++ & - & + \\
- anaplasmose & ++ & ++ & ++ \\
Amblyomma & - & ++ & - & + \\
Maladie transmise : & - & +++ & +++ & + \\
- cowdriose & + dermatophilose & & & \\
\hline
\end{tabular}


Figure 1. Distribution actuelle de la tique Amblyomma variegatum dans les Antilles, et année d'introduction observée ou supposée dans les différentes îles.
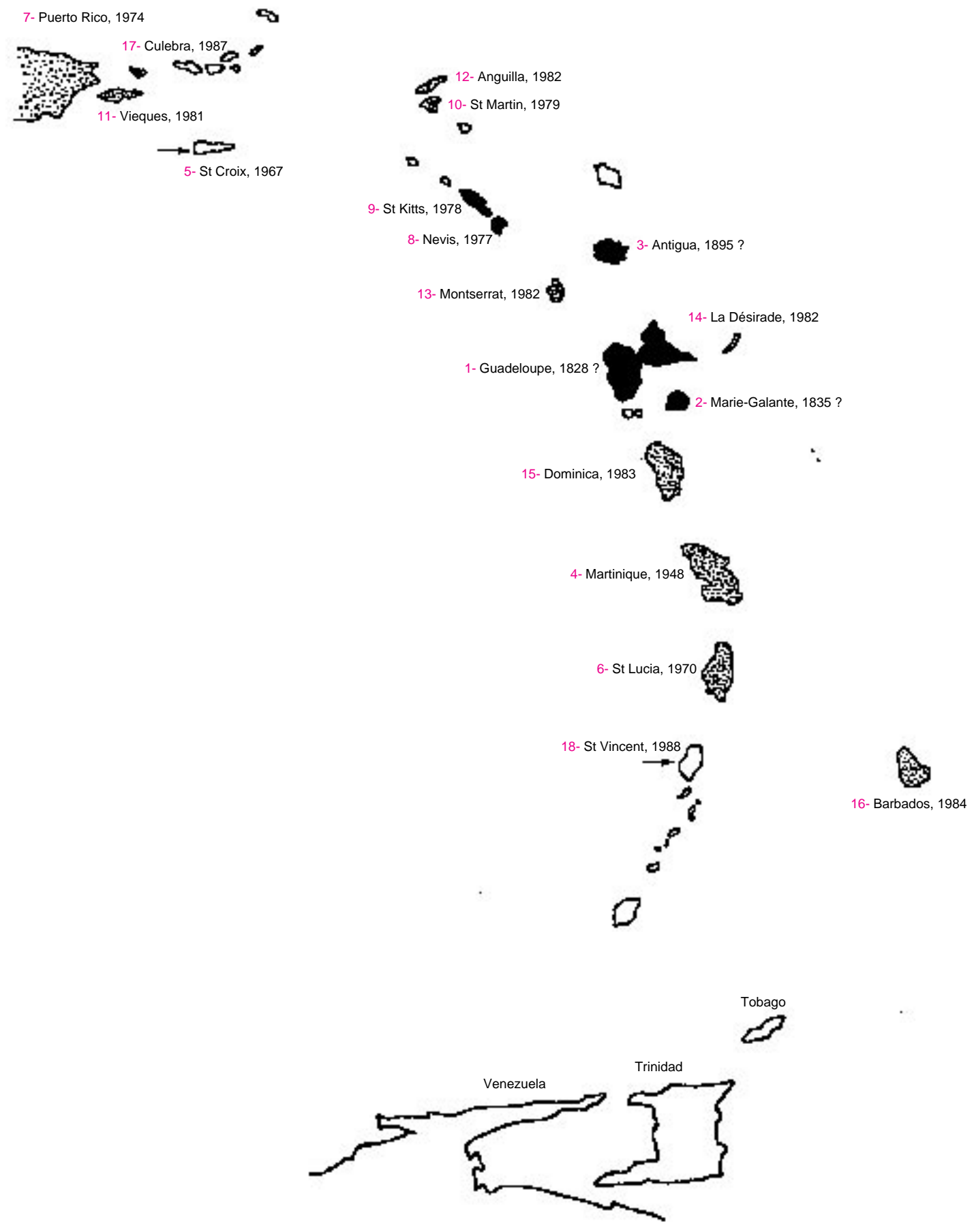

\section{a / Aptitude à la propagation}

Les tiques ont peu de mobilité intrinsèque. Pour les 2 espèces, les femelles gorgées détachées de l'hôte et les larves ont des capacités de déplacement très limitées, de l'ordre de quelques dizaines de centimètres. Nymphes et adultes d'Amblyomma ont une plus grande autonomie, de quelques dizaines de mètres (Barré 1989), insuffisante cependant pour assurer une dissémination efficace de l'espèce à distance moyenne ou grande.

D'autres modes de transport, dans le fumier, la terre, le foin ou l'herbe coupée (infestation du sud de la Martinique par Amblyomma selon ce dernier mode, Morel 1982) peuvent assurer une propagation locale, mais le mode de dissémination le plus efficace consiste dans le déplacement des hôtes parasités (Barré et al 1987). Le risque de propagation est d'autant plus élevé que les hôtes sont plus infestés, qu'ils le sont par des femelles, et que ces hôtes sont abondants et se déplacent souvent et/ou en grand nombre. Le risque est accru lorsque la tique est résistante à tout ou partie des acaricides du commerce. Les mesures judicieuses et rigoureuses de détiquage prises au départ et à l'arrivée de bétail 
peuvent alors s'avérer illusoires et tromper l'importateur sur l'ampleur des risques encourus.

Boophilus ne peut accomplir un développement complet que sur des bovins. Le transport de bovins non détiqués, ou détiqués mais infestés par des souches résistantes aux acaricides, est le mode le plus efficace, sinon exclusif, de propagation de cette espèce. Dans les Petites Antilles, au moins 2 cas récents de résistance de Boophilus aux pyréthrinoïdes ont été décrits : en Martinique (F. RoseRosette, communication personnelle 1995) et à St Kitts (Pegram et al 1996).

Amblyomma produit des tiques viables après le gorgement, le détachement et la mue des immatures ou après le gorgement, le détachement et la ponte des femelles. Tous les hôtes peuvent être des vecteurs de propagation. Les plus efficaces sont ceux qui hébergent des femelles, chaque femelle pouvant engendrer une population de 10 à 30000 larves alors que chaque immature ne produit qu'une autre tique. Les caprins, mais surtout les bovins fortement parasités par les adultes sont donc les propagateurs potentiellement les plus efficaces d'Amblyomma variegatum. Cependant, la réglementation actuelle et les

Figure 2. Nombre cumulé d'îles de la Caraïbe infestées par Amblyomma variegatum et colonisées par le Héron garde-bœufs (d'après Barré et al 1995).

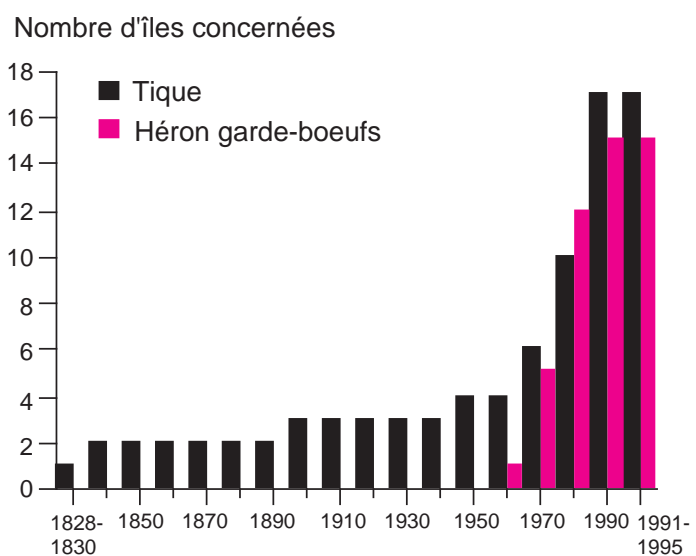

contrôles sanitaires aux frontières limitent considérablement le rôle de ces animaux domestiques dans la propagation régionale. Elle semble aujourd'hui assurée essentiellement par des oiseaux migrateurs ou erratiques tels que le Héron garde-bœufs (Bubulcus ibis), une espèce originaire d'Afrique, arrivée au milieu de ce siècle dans les Petites Antilles et dont l'expansion géographique et démographique est concomitante de l'accélération récente de l'infestation des îles de la Caraïbe (figure 2).

Le rôle potentiel de cet oiseau a été l'objet d'une étude spécifique en Guadeloupe et à Antigua où l'amplitude et la fréquence de ses déplacements inter-îles ont été démontrées (Corn et al 1993), après qu'il eut été reconnu comme un hôte important des immatures d'Amblyomma (Barré et al 1988, Corn et al 1993 ; tableau 3). Cet oiseau est très inféodé aux bovins pour la recherche de sa nourriture, il est présent partout (Arendt 1988) et a, dans toutes les îles des Antilles, des populations importantes composées de plusieurs milliers d'individus par île (2 000 à 15000$)$, dépendant de la taille de l'île (Corn et al 1993, Krebs et al 1993, Barré et al 1995). De plus, une partie de sa population antillaise a un comportement migratoire (5 à $10 \%$ des oiseaux marqués en Guadeloupe et à Antigua : Corn et al 1993) et effectue des déplacements saisonniers assurant des brassages de population et permettant des transferts de parasites dans la très vaste zone d'extension de l'oiseau qui va du Canada à l'Argentine.

\section{b / Capacité d'implantation}

L'aire de répartition des deux tiques dépend de leurs exigences bioclimatiques. Boophilus est déjà établi dans toutes les îles de la Caraïbe et sur le continent américain, du nord du Mexique au nord de l'Argentine. Cette tique a sans doute atteint toute son aire d'extension potentielle. Il n'en est pas de même d'Amblyomma qui n'est encore présent que dans les Petites Antilles et à Porto Rico, mais qui s'acclimaterait parfaitement dans les zones d'élevage sur prairies et savanes et dans les clairières et en lisière de forêt à basse et moyenne altitude, depuis le sud du
La propagation des tiques par les animaux d'élevage est limitée par les contrôles sanitaires aux frontières; elle se fait surtout par les oiseaux migrateurs pour Amblyomma.

Tableau 3. Infestation des hôtes par Boophilus et Amblyomma. Résultat de bilans parasitaires faits en Guadeloupe : pourcentage moyen d'animaux infestés (\% inf), nombre moyen de larves (La), de nymphes (Ny) et d'adultes (Ad) par animal. Vingt Rats noirs, 48 souris et 256 oiseaux migrateurs (Charadriidés) n'étaient pas infestés (en partie d'après Barré et al 1988).

\begin{tabular}{|l|r|c|c|c|c|c|c|c|c|}
\hline & & \multicolumn{4}{|c|}{ Boophilus Microplus } & \multicolumn{3}{c|}{ AmblyommaVariegatum } \\
\hline Hôte & $\mathrm{n}$ & $\%$ inf & $\mathrm{La}$ & $\mathrm{Ny}$ & $\mathrm{Ad}$ & $\%$ inf & $\mathrm{La}$ & $\mathrm{Ny}$ & $\mathrm{Ad}$ \\
\hline Bovin & 11 & 45,4 & 8,5 & 11,8 & 5,4 & 100 & 265,9 & 35,8 & 43,1 \\
Caprin & 12 & & & & & 50 & 547,2 & 12,1 & 17,6 \\
Chien & 10 & 20 & 0,8 & & & 80 & 103,5 & 7,5 & \\
Mangouste & 123 & 0,8 & 0,01 & & & 51,2 & 4 & 0,3 & \\
Poulet & 6 & & & & & 66,7 & 5,3 & 0,2 & \\
Héron garde-bœufs & 80 & 1,2 & 0,02 & & & 26,2 & 1,7 & 0,05 & \\
Oiseaux divers & 120 & & & & & 25,8 & 0,7 & 0,1 & \\
\hline
\end{tabular}


Mexique jusqu'au sud du Brésil et dans toutes les Grandes Antilles (figure 3). Cette région climatique tropicale correspond à une zone où la tique rencontre des conditions compatibles avec ses exigences thermiques (températures moyennes mensuelles comprises entre 20 et $30{ }^{\circ} \mathrm{C}$ ) et hygrométriques (humidité relative entre 75 et $95 \%$, pluviométrie de 500 à 2750 $\mathrm{mm})$.

Dans toute cette région, l'implantation sera facilitée si les hôtes favorables aux adultes que sont les ruminants de grande taille, qu'ils soient domestiques ou sauvages (cervidés américains notamment), sont accessibles et abondants.

\section{c / Capacité de développement de la population}

Bien qu'Amblyomma variegatum ait une grande fécondité (10 à 30000 œufs par femelle) comparée à Boophilus microplus (2 500 œufs), la nécessité de trouver 3 hôtes au cours de son cycle contre un seul pour Boophilus la handicape fortement en terme de nombre de générations annuelles possibles et de nombre de descendants. Ainsi, en supposant que les 3 stades d'Amblyomma et les larves de Boophilus mettent chacun 30 jours à trouver un hôte, et compte tenu des durées d'accomplissement des diverses phases vitales

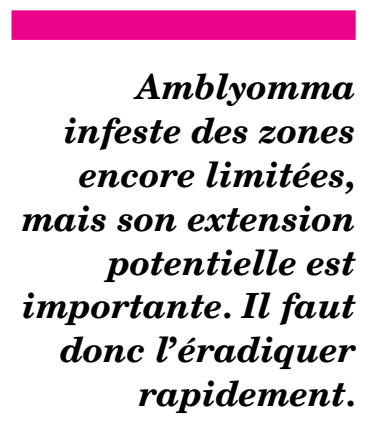

Figure 3. Aire de distribution actuelle et potentielle de la tique Amblyomma variegatum dans l'hémisphère occidental.

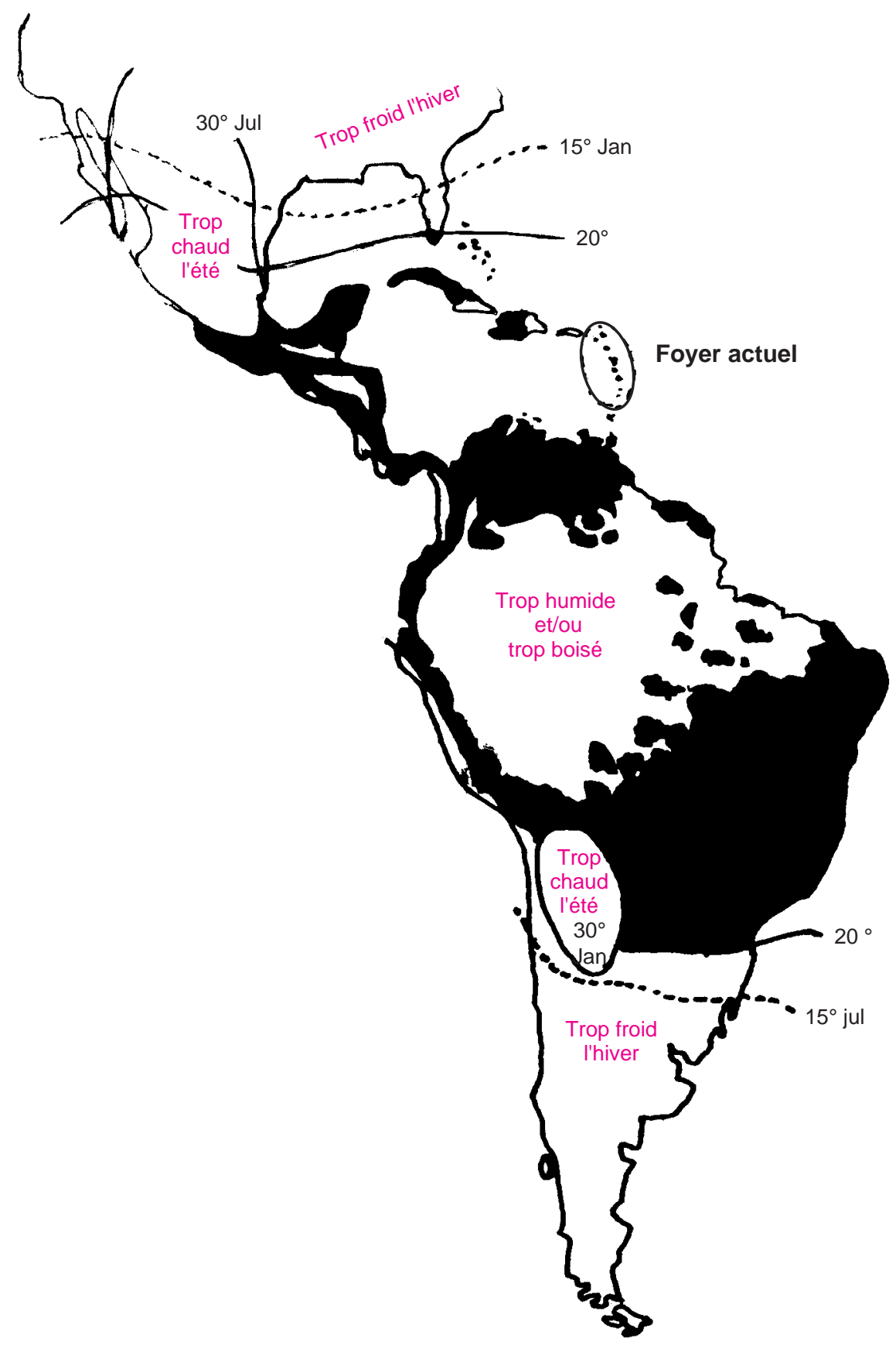


à $25{ }^{\circ} \mathrm{C}$ (repas de sang, mue, oviposition, incubation des œufs...), le cycle d'Amblyomma va durer 232 jours, celui de Boophilus seulement 93 jours. Dans les conditions de température moyennes des Antilles, Amblyomma pourra donc théoriquement produire 3 générations en 2 ans contre 8 pour Boophilus et engendrer $10^{12}$ descendants alors que Boophilus en aura produit $10^{25}$ (10 milliards de fois plus).

Cette extrême productivité numérique de Boophilus explique ses grandes capacités de colonisation et la grande difficulté de son éradication dans ses zones de plus grand confort climatique où elle a 4 à 5 générations annuelles. Cette tique a par exemple été éradiquée de Porto Rico vers 1960, mais l'a presque entièrement reconquis 2 ans après sa réintroduction accidentelle en 1978 (Combs 1989), alors que Amblyomma, qui est arrivé en Martinique en 1948, ne l'a encore qu'à moitié colonisée. Cette caractéristique d'Amblyomma laisse à penser, que dans des zones bien circonscrites, une action de lutte soutenue et systématique, généralisée à tout le cheptel, hôte potentiel des tiques adultes, pourrait aboutir à l'éradication de ses populations.

\section{3 / Conséquences pour la définition des principes de lutte contre les tiques aux Antilles}

Toutes les Antilles et toutes les zones continentales favorables à Boophilus sont infestées par cette tique. Une action d'éradication dans les Antilles aurait peu de chances de succès et de pérennité compte tenu de son extrême prolificité dans les Antilles - à l'optimum de son aire de confort climatique, des risques de réinfestation à partir de zones voisines restées infestées et de sa propension à développer des résistances aux acaricides.

Le contrôle de cette espèce doit faire appel aux principes de la lutte intégrée, combinant le recours à des animaux résistants à l'infestation et la pratique de la lutte agronomique, et minimisant l'emploi des acaricides. Ceci est possible avec le bétail créole et zébu, largement dominant aux Antilles, naturellement résistant aux tiques et aux maladies transmises (babésioses et anaplasmose) et pour lequel des infestations modestes sont sans conséquences directes sur l'état de santé. Avec du bétail taurin européen, la préservation d'une population permanente minimale de tiques infectantes (6 à 8 femelles gorgées par animal) est même indispensable au maintien de l'équilibre enzootique vis-à-vis des maladies transmises. C'est ce que Morel (cité par Troncy et al 1981) appelle le paradoxe épidémiologique.

A la différence de Boophilus, les zones infestées par Amblyomma sont encore de superficie relativement limitée, elles sont isolées et éloignées de la source africaine d'ori- gine, permettant de penser que les risques de réinfestation de territoires assainis seraient insignifiants.

Notons que la propagation d'Amblyomma est insidieuse puisque les immatures peuvent être transportés par des animaux sauvages de petite taille comme des oiseaux migrateurs. Cette tique peut s'établir ensuite au stade adulte sur des animaux de plus grande taille comme les ongulés, éventuellement sauvages, échappant ainsi à l'attention des éleveurs et des services vétérinaires. Ces animaux sauvages peuvent donc théoriquement entretenir un cycle occulte pendant une longue période avant que la tique n'atteigne des animaux domestiques, ne soit reconnue et identifiée puis l'objet de mesures de contrôle. Par ailleurs, les exigences écologiques et climatiques d'Amblyomma sont étendues, ce qui la prédispose à coloniser de vastes territoires dans la zone intertropicale du Nouveau Monde. Ces éléments permettent de justifier la nécessité d'intervenir rapidement et de tenter de l'éradiquer pendant que sa zone d'extension est encore bien circonscrite aux Antilles. Cette nécessité est renforcée par la connaissance de l'extrême pathogénicité de cette tique, résultant en particulier de sa capacité à favoriser les formes cliniques sévères, épizootiques et mortelles de la dermatophilose. Aux Antilles, son impact économique s'est révélé avec toute son acuité dans les zones récemment infestées (Uilenberg et al 1984).

Cette intervention est jugée faisable sur Amblyomma aux Antilles, en raison notamment du passage obligé, au stade adulte, par des animaux domestiques (bovins, équins, petits ruminants) qui, en général, peuvent être contenus et traités. La faible productivité et faculté de diffusion locale de cette tique a également été en faveur de la mise en œuvre de cette stratégie. La lutte chimique intensive ne semble pas devoir être compromise par le phénomène de résistance aux acaricides qui n'a pas encore été mis en évidence pour Amblyomma variegatum aux Antilles (et qui est exceptionnel ailleurs dans le monde pour cette espèce). Elle reste sensible aux acaricides du commerce (Garris et Barré 1990 et résultats non publiés). Ainsi, un programme d'éradication d'Amblyomma variegatum a été proposé et soutenu par divers partenaires politiques, économiques et scientifiques de la région (FAO 1992). Il est opérationnel en Guadeloupe et Martinique depuis 1994, grâce notamment à une participation financière de l'Etat, de la CEE et des collectivités locales, ainsi que dans certaines îles anglophones du nord des Petites Antilles (Anguilla, St Kitts, Nevis) où le programme est coordonné par la FAO (Pegram et al 1996).

Les principes, la stratégie et les conditions de succès de cette éradication : hôtes à traiter, rythme et durée du traitement, choix des acaricides et des procédés d'application, organisation à mettre en place, nécessité d'une action régionale globale... ont été définis par ailleurs (Barré et Garris 1990, FAO 1992, Barré et al

\section{Boophilus infeste déjà les Antilles et une partie du continent américain ce qui rend difficile son éradication, d'autant plus qu'elle développe une résistance aux acaricides.}


1996) et ont été adoptés avec quelques nuances dans toutes les Antilles françaises et anglophones :

- campagnes si possible simultanées dans les différentes îles infestées, ou au moins sans grand décalage de temps ;

- coordination des interventions par un Comité de pilotage ;

- programme sur 5 ans : 1 année de préparation (information, communication, appels d'offres, recrutement des agents, constitution des secteurs et tournées de détiquage...), 3 années de traitement intensif en continu (pour tenir compte de la durée de survie maximale des adultes libres : 2 ans), 1 année de surveillance, pour repérer et traiter les poches résiduelles d'infestation.

Dans ce laps de temps :

- recensement et détiquage de tous les bovins, chevaux, petits ruminants, porcs à l'attache et chiens, hôtes potentiels des tiques adultes. Action concomitante de décanisation ;

- détiquage toutes les 2 semaines (pour tenir compte de la durée d'efficacité résiduelle des acaricides, du délai de production de phéromones par les mâles ( 5 jours) et de gorgement des femelles ( 7 à 15 jours), à jour fixe par une équipe mobile (tank et pompe sur véhicule ou topique prêt à l'emploi), de tous les hôtes potentiels des adultes pendant 3 ans ;

- utilisation de pyréthrinoïdes (fluméthrine, deltaméthrine) ou de triazapentadiènes (amitraze) ;

- détiquage par aspersion, ou mieux et si possible, administration de pyréthrinoïdes en topique dorsal (mais le coût très supérieur ne permet pas toujours un emploi généralisé) ;

- contrôle de l'efficacité de la campagne par suivi régulier de l'évolution des populations de tiques sur les animaux et de la prévalence des pathologies associées ; contrôle de la persistance de la stabilité enzootique vis-à-vis des babésioses.

Dans les Antilles françaises, la campagne a été lancée en 1994. Elle a connu quelques difficultés en Guadeloupe en 1996, mais devrait reprendre en 1997. Son succès dans ces îles dépendra de la rigueur avec laquelle elle sera menée. Son échec conduirait inéluctablement à l'installation de la tique sur le continent, ainsi qu'à celle des graves maladies qui lui sont associées et, dans la Caraïbe, à la nécessité d'une lutte coûteuse et permanente contre un des principaux freins au développement de l'élevage.

\section{Références bibliographiques}

Arendt W.J., 1988. Range expansion of Cattle Egret (Bubulcus ibis) in the Greater Caribbean Basin. Colonial Waterbirds, 11, 252-262.

Barré N., 1989. Biologie et écologie de la tique Amblyomma variegatum (Acarina : Ixodina) en Guadeloupe (Antilles françaises). Thèse Doc. Etat. ParisSud, Orsay, $267 \mathrm{p}$.

Barré N., Garris G.I., 1990. Biology and ecology of Amblyomma variegatum (Acari : Ixodidae) in the Caribbean : implications for a regional eradication program. J. Agric. Entomol., 7, 1-9.

Barré N., Uilenberg G., Morel P.C., Camus. E., 1987. Danger of introducing heartwater onto the American mainland : potential role of indigenous and exotic Amblyomma ticks. Onderstepoort. J. Vet. Res., 54, 405-417.

Barré N., Garris G.I., Borel G., Camus E., 1988. Hosts and population dynamics of Amblyomma variegatum (Acari : Ixodidae) on Guadeloupe, French West Indies. J. Med. Entomol., 25, 111-115.

Barré N., Garris G.I., Camus E., 1995. Propagation of the tick Amblyomma variegatum in the Caribbean. Rev. Sci. Tech. Off. Int. Epiz., 14, 841-855.

Barré N., Camus E., Fifi J., Fourgeaud P., Numa G., Rose-Rosette F., Borel H., 1996. Tropical Bont Tick eradication campaign in the French Antilles : current status. Ann. N. Y. Acad. Sci., 791, 64-76.

Camus E., 1987. Contribution à l'étude épidémiologique de la cowdriose (Cowdria ruminantium) en Guadeloupe. Thèse Doc. Etat. Paris-Sud, Orsay, 201 p.
Champanhet F., 1995. La filière viande bovine à la Martinique, chiffres et tendances 1994. Cemagref Martinique, note $\mathrm{n}^{\circ} 27,1-4$

Combs G.P., 1989. Economics of tick eradication in Puerto-Rico. In : The eradication of ticks. FAO Animal Production and Health Paper $n^{\circ} 75$, FAO, Rome, 91-98.

Corn J., Barré N., Thiébot B., Creekmore T.E., Garris G.I., Nettles V.F., 1993. The potential role of cattle egrets, Bubulcus ibis, (Ciconiformes : Ardeiae) in the dissemination of Amblyomma variegatum (Acari : Ixodidae) in the Eastern Caribbean. J. Med. Entomol., 6, 1029-1037.

Curasson G., 1943. Trypanosoma vivax et variétés. Traité de Protozoologie humaine et comparée. Tome I, 267-269. Editions Vigot, Paris.

FAO, 1992 Programme for the eradication of Amblyomma variegatum from the Caribbean. Report, 22 p. +9 Annexes.

Garris G.I., Barré N., 1990. Acaricide susceptibility of Amblyomma variegatum (Acari : Ixodidae) from Puerto Rico and Guadeloupe. Exp. Appl. Acarol., 12, 171-179.

Krebs E A., Riven-Ramsey D., Hunte W., 1993. The colonization of Barbados by the cattle egret (Bubulcus ibis), 1956-1990. Colonial Waterbirds, 17, 86-90.

Maillard N., Maillard J.C., 1995. Historique du peuplement bovin dans les îles françaises des Antilles. Synthèse bibliographique. Historiae Medicinae Veterinariae, (sous presse).

Morel P.C., 1966. Etude sur les tiques du bétail en Guadeloupe et Martinique. I. Les tiques et leur distribution. (Acariens, Ixodidae). Rev. Elev. Méd. Vét. Pays Trop., 19, 307-321. 
Morel P.C., 1982. Enquête sur le programme de lutte contre les tiques du bétail en Martinique (juin 1982). Propositions pour un nouveau programme. IEMVT, Maisons-Alfort, France, polycopié, 17 p.

Nellis D.W., Everard C.O.R., 1983. The biology of the mongoose in the Caribbean. Studies on the fauna of Curaçao and other Caribbean islands, 64, $162 \mathrm{p}$.

Pegram R.G., Rota A., Sebrecht M.T., 1996. Amblyomma Program Council, Annual APC Report, 1995-1996, 39 p.

Salas M., Planchenault D., Roy F., 1988. Etude des systèmes d'élevage bovin traditionnel en Guadeloupe, Antilles françaises. Résultat d'enquêtes. Rev. Elev. Méd. Vét. Pays Trop., 41, 197-207.

SCEES, 1990. Recensement Général de l'Agriculture, 1988-1989, Guadeloupe. Agreste. La Statistique agricole, $\mathrm{n}^{\circ} 2,104 \mathrm{p}$. Ministère de l'Agriculture et de la Forêt, Paris.
Stachurski F., 1988. Impact économique actuel et intérêt de l'éradication de la tique Amblyomma variegatum pour l'élevage bovin guadeloupéen. Thèse Doc. vét. Créteil, $126 \mathrm{p}$.

Tatareau J.C., Lalaus G., Pensedent-Erblon J., Shitalou E., Milhet P., Barré N., Matheron G., 1991. L'élevage des petits ruminants en Martinique, Guadeloupe et Guyane : situation actuelle. Rev. Elev. Méd. Vét. Pays Trop., ${ }^{\circ}$ spécial, 45-50.

Troncy P.M., Itard J., Morel P.C., 1981. Manuels et Précis d'Elevage : 10 - Précis de Parasitologie vétérinaire tropicale. Ministère de la Coopération et du développement/Institut d'Elevage et de Médecine vétérinaire des Pays tropicaux, $717 \mathrm{p}$.

Uilenberg G., 1990. Extension de la tique Amblyomma variegatum dans les Antilles : comment expliquer cette grave menace et que faire ? Rev. Elev. Méd. Vét. Pays Trop., 43, 297-299.

Uilenberg G., Barré, N., Camus E., Burridge M.J., Garris G.I., 1984. Heartwater in the Caribbean. Prev. Vet. Med., 2, 255-267.
The ticks of the ruminants of the Lesser Antilles : their biology, their economic importance and defensive strategies.

Two species of tropical ticks, Boophilus microplus, originating from Asia and Amblyomma variegatum originating from Africa, parasitize the ruminants of the West Indies and in particular the French Antilles (Guadeloupe and Martinique). They are the vectors of various diseases including babesiosis and anaplasmosis for B. microplus and benign theileriosis and cowdriosis for A. variegatum. In addition, A. variegatum is associated with clinically severe and often fatal forms of Dermatophus congolensis disease (cutaneous Streptothricosis). The majority of the cattle on Guadeloupe are the Creole type, which are highly resistant to these diseases. The goats on Guadeloupe, however, suffer heavily from cowdriosis. The overall economic damage due to ticks on Guadeloupe is estimated to be $13.8 \mathrm{MF}$. While B. microplus seems to cover the full extent of its potential geographic range including the West Indies and the continent, A. variegatum, which is the more pathogenic species, is only found in the lesser Antilles and Porto Rico. Around the middle of the century it was only found on 4 islands, but over the last 25 years it has infested 14 new islands. It seems highly likely that cattle egrets are to blame for the recent acceleration in its propagation. These egrets came to the Lesser Antilles from Africa near the end of the 50's, and are currently found throughout the Caribbean. The A. variegatum infestation now threatens the American continent and the Greater Antilles. Despite its great capacity for regional distribution via its hosts, this tick has a limited productivity and is therefore less able to install itself and form colonies than $B$. microplus. It is therefore relatively easy to eradicate a colony if proper counter measures are taken as soon as it is detected. Wherever such a colony is established, taking rigorous measures that interrupt the development cycle should destroy the population. In addition, $A$. variegatum does not seem to be resistant to acaricides and seems to be relatively specific to domestic animals of a large size during its adult stage. These aspects favour the development of a defensive strategy which would eradicate these ticks by regularly applying acaricides on their domestic hosts.

BARRÉ N., 1997. Les tiques des ruminants dans les Petites Antilles : biologie, importance économique, principes de lutte. INRA Prod. Anim., 10 (1), 111-119. 